ARTICLE

\title{
Low thermal conductivity of iron-silicon alloys at Earth's core conditions with implications for the geodynamo
}

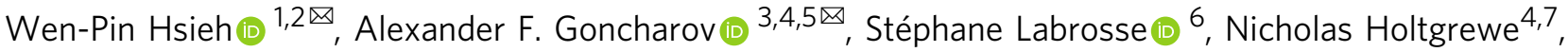
Sergey S. Lobanov (1) ${ }^{4,8}$, Irina Chuvashova ${ }^{4}$, Frédéric Deschamps ${ }^{1} \&$ Jung-Fu Lin (i) ${ }^{9 凶}$

Earth's core is composed of iron ( $\mathrm{Fe}$ ) alloyed with light elements, e.g., silicon ( $\mathrm{Si}$ ). Its thermal conductivity critically affects Earth's thermal structure, evolution, and dynamics, as it controls the magnitude of thermal and compositional sources required to sustain a geodynamo over Earth's history. Here we directly measured thermal conductivities of solid Fe and Fe-Si alloys up to $144 \mathrm{GPa}$ and $3300 \mathrm{~K} .15$ at\% Si alloyed in Fe substantially reduces its conductivity by about 2 folds at $132 \mathrm{GPa}$ and $3000 \mathrm{~K}$. An outer core with 15 at\% Si would have a conductivity of about $20 \mathrm{~W} \mathrm{~m}^{-1} \mathrm{~K}^{-1}$, lower than pure $\mathrm{Fe}$ at similar pressure-temperature conditions. This suggests a lower minimum heat flow, around 3 TW, across the core-mantle boundary than previously expected, and thus less thermal energy needed to operate the geodynamo. Our results provide key constraints on inner core age that could be older than two billion-years.

\footnotetext{
${ }^{1}$ Institute of Earth Sciences, Academia Sinica, Nankang, Taipei 11529, Taiwan. ${ }^{2}$ Department of Geosciences, National Taiwan University, Taipei 10617, Taiwan. ${ }^{3}$ Key Laboratory of Materials Physics, Institute of Solid State Physics CAS, Hefei 230031, China. ${ }^{4}$ Earth and Planets Laboratory, Carnegie Institution of Washington, Washington, DC 20015, USA. ${ }^{5}$ Institut de Physique du Globe de Paris, Université de Paris, Paris 75005, France. ${ }^{6}$ Univ Lyon, ENSL, Univ Lyon 1, CNRS, LGL-TPE, F-69007, Lyon, France. ${ }^{7}$ Department of Mathematics, Howard University, Washington, DC 20059, USA. ${ }^{8}$ GFZ German Research Center for Geosciences, Telegrafenberg, 14473 Potsdam, Germany. ${ }^{9}$ Department of Geological Sciences, Jackson School of Geosciences, University of Texas at

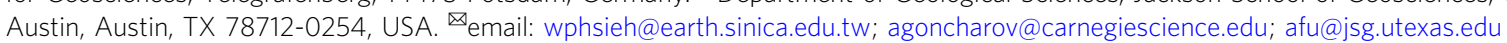


T hermal conductivity in Earth's core plays a fundamental role in controlling the dynamics and evolution of this region $^{1}$. Core convection and the resulting geodynamo are predominantly driven by thermal and compositional sources ${ }^{2-4}$. Energy and entropy balances of the core indicate that a convective geodynamo requires a minimum core-mantle boundary (CMB) heat flow to operate, where the minimum value increases with increasing core thermal conductivity. If the core thermal conductivity is low enough, purely thermal convection may have sustained a geodynamo for the entire Earth history. By contrast, if thermal conductivity of the core is high, the isentropic heat flux across $\mathrm{CMB}$ is high and compositional convection, which lowers the value of the critical CMB heat flow, is needed to sustain a geodynamo ${ }^{5-9}$. For the generation of most recent magnetic fields, crystallization of the inner core provides a substantial latent heat and compositional source allowing geodynamo to operate even at a high core thermal conductivity ${ }^{5}$. Precipitation and transport of light elements, e.g., $\mathrm{Si}, \mathrm{O}, \mathrm{Mg}$, etc., from the outer core to the lowermost mantle have also been proposed as possible mechanisms to run a geodynamo in ancient Earth $\sim 3.4 \mathrm{Gyr} \mathrm{ago}^{6-9}$, before the inner core started to grow. Core thermal conductivity, influenced by its exact composition and temperature over its history, thus holds a key to decipher the enigmatic thermal and compositional evolutions of Earth's core, providing important insights into the origin and history of palaeomagnetic fields, available thermal vs. compositional energy sources for driving the geodynamo, and age and growth rate of the inner core ${ }^{10,11}$.

In the past decades, geophysical and geochemical observations have revealed density deficits in Earth's inner and outer cores. Comparison between seismic models and the density of pure $\mathrm{Fe}$ at relevant core pressure $(P)$-temperature $(T)$ conditions suggests that a certain amount of light elements alloyed with $\mathrm{Fe}$ is present in the core ${ }^{1,12-14}$. Among candidate light elements, $\mathrm{Si}$ is a likely candidate with $\sim 8$ and $4 \mathrm{wt} \%(\approx 15$ and 7 at $\%)$ in the outer and inner cores, respectively, due to its geophysical and geochemical characteristics $1,12-14$. Other light elements such as $\mathrm{O}, \mathrm{S}, \mathrm{C}$, or $\mathrm{H}$ could also exist in the core with Si. Moreover, at high $P-T$ conditions relevant to the core, $\mathrm{Fe}-\mathrm{Si}$ alloy is stable in hexagonal close-packed (hcp) structure as $\mathrm{Si}$ is readily dissolved in $\mathrm{Fe}$, and its physical properties, e.g., sound velocities and density, are able to account for the seismic data observed in the core $e^{1,12-14}$. These features motivate us to use $\mathrm{Fe}-\mathrm{Si}$ alloy as a representative to investigate effects of light elements on the thermal conductivity of $\mathrm{Fe}$ in the core and to access the importance of various energy sources for the geodynamo.

There are two major mechanisms of heat transfer, i.e., thermal conduction and convection, in the core, where the thermal radiation mechanism does not effectively transfer heat in metallic Fe and Fe-rich alloys. Though thermal conduction of Fe alloyed with light elements at core conditions is essential to reconstruct thermal history of the core and geodymano, it has never been directly measured at relevant high $P-T$ conditions. Previous theoretical calculations have predicted a highly thermally conductive core with a thermal conductivity of about $80-200 \mathrm{~W} \mathrm{~m}^{-1}$ $\mathrm{K}^{-1}$ at the outer core and $150-300 \mathrm{~W} \mathrm{~m}^{-1} \mathrm{~K}^{-1}$ at the inner core, respectively ${ }^{15-18}$. These results, however, are difficult to reconcile with observations of early magnetic fields ${ }^{11,19}$ because these high conductivity values suggest a young inner core and require either a very hot initial core ${ }^{10}$ or alternative buoyancy sources in the form of light element extraction from the top of the core ${ }^{6-8}$ to explain the ancient dynamo. Recent studies ${ }^{20,21}$ on the pure Fe thermal conductivity at the outermost core conditions using two different experimental approaches show a large discrepancy: a high value of about $226 \mathrm{~W} \mathrm{~m}^{-1} \mathrm{~K}^{-1}$ was inferred from the electrical resistivity data ${ }^{21}$, while a low value of about $33 \mathrm{~W} \mathrm{~m}^{-1} \mathrm{~K}^{-1}$ was obtained by measurements using transient heating $(\mathrm{TH})$ laser technique ${ }^{20}$. These results led to contradictory implications for the age and heat flow budget of the core. Prior estimates of core thermal conductivity from experiments largely focused on converting electrical resistivity of $\mathrm{Fe}$ and $\mathrm{Fe}$ alloys at high $P-T$ conditions into thermal conductivity via the Wiedemann-Franz (WF) law with ideal Lorenz number ${ }^{21-27}$, while the validity of WF law at high $P-T$ conditions remains uncertain ${ }^{15}$. As a result, direct and precise thermal conductivity measurements on $\mathrm{Fe}$ alloyed with a major light element at relevant high $P-T$ conditions are critically needed to pin down core's thermal conductivity and to correctly describe the core evolution and dynamics.

In this paper, we showed that the thermal conductivity of $\mathrm{Fe}$ alloyed with 15 at $\% \mathrm{Si}$ is approximately half of the pure $\mathrm{Fe}$ at outer core conditions. This suggests that Earth's geodynamo could be operated by pure thermal convection and that the age of inner core could be older than two billion-years.

\section{Results}

Thermal conductivity at high pressure and room temperature. We combined ultrafast time-domain thermoreflectance (TDTR) with diamond anvil cell (DAC) technique to precisely measure the thermal conductivity of both single-crystal and powder samples of pure $\mathrm{Fe}$ and powder of $\mathrm{Fe}_{1-\mathrm{X}} \mathrm{Si}_{\mathrm{x}}(x=0.04$ and 0.15$)$ alloys to $120 \mathrm{GPa}$ at room temperature. TDTR is a well-developed ultrafast metrology that uses sub-picosecond optical pulses to pump and probe thermal transport through the sample, providing high-precision thermal conductivity measurements at pressures over $100 \mathrm{GPa}^{28,29}$ (Methods). The thermal conductivity of bodycentered cubic (bcc) Fe (black symbols in Fig. 1) at ambient conditions is $\approx 76 \mathrm{~W} \mathrm{~m}^{-1} \mathrm{~K}^{-1}$. Upon compression, the thermal conductivity increases with pressure, while drastically decreases at $P \approx 13 \mathrm{GPa}$ due to the structural transition from bcc to hcp phase, where the enhanced electron correlation reduces lifetimes of quasiparticles and thus decreases the thermal conductivity ${ }^{30}$. Interestingly, the pressure dependence of thermal conductivity shows a minimum around $40 \mathrm{GPa}$, which may be associated with an electronic topological transition ${ }^{31}$, and then increases again with pressure, reaching $\approx 120-130 \mathrm{~W} \mathrm{~m}^{-1} \mathrm{~K}^{-1}$ near the CMB pressures.

Compared with pure $\mathrm{Fe}$, the thermal conductivity of $\mathrm{Fe}_{0.96} \mathrm{Si}_{0.04}$ alloy (blue symbols in Fig. 1) at ambient conditions is significantly reduced to $16.5 \mathrm{~W} \mathrm{~m}^{-1} \mathrm{~K}^{-1}$, much lower than the previously estimated light element effects ${ }^{17,20,24}$. Upon compression, the thermal conductivity increases slowly until $P \approx 40 \mathrm{GPa}$, after which it saturates and remains at $\approx 40 \mathrm{~W} \mathrm{~m}^{-1} \mathrm{~K}^{-1}$ to around $110 \mathrm{GPa}$, i.e., a factor of 3 smaller than the pure hcp-Fe at similar pressures. Moreover, addition of 15 at $\%$ Si impurity further decreases the thermal conductivity of hcp- $\mathrm{Fe}_{0.96} \mathrm{Si}_{0.04}$ at high pressure (see $\mathrm{Fe}_{0.85} \mathrm{Si}_{0.15}$, red symbols in Fig. 1). At ambient conditions the thermal conductivity starts from an even lower value of $11.5 \mathrm{~W} \mathrm{~m}^{-1} \mathrm{~K}^{-1}$; similar to $\mathrm{Fe}_{0.96} \mathrm{Si}_{0.04}$, it increases slowly with pressure, while saturates to $\approx 19 \mathrm{~W} \mathrm{~m}^{-1} \mathrm{~K}^{-1}$ between $P \approx 35-120 \mathrm{GPa}, \sim 6-7$ fold smaller than the pure hcp-Fe. We note that alloying 4 and 15 at\% ( $\approx 2$ and $8 \mathrm{wt} \%$, respectively) $\mathrm{Si}$ in $\mathrm{Fe}$ substantially changes the pressure dependence of thermal conductivity (i.e., the concave behavior around $40 \mathrm{GPa}$ was only observed in pure hcp-Fe, not in $\mathrm{Fe}-\mathrm{Si}$ alloys), suggesting that even small Si doping may stabilize the topology of the Fermi surface of hcp-Fe under compression. The substantial suppression of the thermal conductivity with the addition of 4 and $15 \mathrm{at} \% \mathrm{Si}$ in $\mathrm{Fe}$ is presumably due to the strongly inelastic electron-impurity scattering $22,25,27$.

Thermal conductivity at high pressure-temperature conditions. To constrain the combined effects of silicon alloying and 


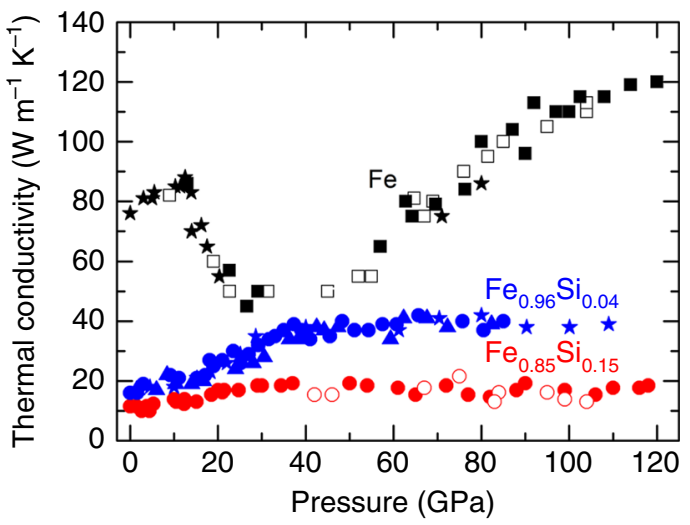

Fig. 1 Thermal conductivity at high pressure and room temperature. The thermal conductivity of powder Fe (black squares) is comparable with that of single-crystal Fe (black stars) and much larger than that of powder $\mathrm{Fe}_{0.96} \mathrm{Si}_{0.04}$ (blue symbols) and $\mathrm{Fe}_{0.85} \mathrm{Si}_{0.15}$ (red circles), indicating the strong alloying effect of silicon on the thermal conductivity of Fe. Each set of data includes several runs of measurement with solid symbols for compression and open symbols for decompression cycle, respectively. The measurement uncertainties are $\approx 10 \%$ before $30 \mathrm{GPa}, \approx 20 \%$ at $60 \mathrm{GPa}$, and $\approx 25 \%$ at $120 \mathrm{GPa}$. The drastic decrease in the thermal conductivity of Fe around $13 \mathrm{GPa}$ results from the bcc-hcp structural transition ${ }^{30}$.

high temperature, we employed the TH laser technique to measure the thermal conductivity of $\mathrm{Fe}_{1-\mathrm{x}} \mathrm{Si}_{\mathrm{x}}(x=0.04,0.07$, and 0.15$)$ at high $P-T$ conditions. The TH method is a well-developed pulsed laser technique to measure thermal conductivity at simultaneously high $P-T$ conditions ${ }^{20,32}$, where the heat pulses across the sample are probed temporally and spatially using in situ time-domain thermoradiometry, and the thermal conductivity is deduced from the results of the model finite-element (FE) calculations (Methods). Figure 2 shows the thermal conductivity of polycrystalline $\mathrm{Fe}-\mathrm{Si}$ alloys to $144 \mathrm{GPa}$ at $2050-3300 \mathrm{~K}$. Considering the measurement uncertainty, the thermal conductivities of hcp- $\mathrm{Fe}_{0.96} \mathrm{Si}_{0.04}$ and $\mathrm{hcp}-\mathrm{Fe}_{0.93} \mathrm{Si}_{0.07}$ (magenta dotted circles and brown dotted squares, respectively) below $\sim 110 \mathrm{GPa}$ are comparable with the pure hcp- $\mathrm{Fe}^{20}$. We should note that the large scatter in the literature for pure $\mathrm{Fe}$ data ${ }^{20}$ around $40-90 \mathrm{GPa}$ was assigned to be partially associated with the presence of $\gamma$ phase which could affect some of its results at high $P-T$ conditions; however, the $\gamma$-Fe disappears above $100 \mathrm{GPa}$, so the data scatter less in this regime. The thermal conductivity of $\mathrm{Fe}_{0.85} \mathrm{Si}_{0.15}$ (red circles), on the other hand, is slightly smaller than the pure hcp-Fe below $100 \mathrm{GPa}$, though their differences are within uncertainties. Importantly, the thermal conductivity of $\mathrm{Fe}_{0.85} \mathrm{Si}_{0.15}$ decreases significantly with increasing pressure from $~ 120$ to $144 \mathrm{GPa}$. Furthermore, unlike pure hcp-Fe whose thermal conductivity decreases with increasing temperature (Supplementary Fig. 1), the thermal conductivity of hcp$\mathrm{Fe}_{0.96} \mathrm{Si}_{0.04}$ at high temperatures is comparable or slightly larger than that at $300 \mathrm{~K}$ (Supplementary Fig. 2). Moreover, the thermal conductivity of $\mathrm{Fe}_{0.85} \mathrm{Si}_{0.15}$ at high temperatures is generally larger than at $300 \mathrm{~K}$, except at the highest pressures where they become very close to each other (Supplementary Figs. 3 and 4). It is worth noting that at the high $P-T$ conditions of our TH experiments, $\mathrm{Fe}_{0.96} \mathrm{Si}_{0.04}$ stabilizes in the hcp phase ${ }^{33}$ (Supplementary Fig. 5), whereas $\mathrm{Fe}_{0.85} \mathrm{Si}_{0.15}$ almost exclusively falls into the mixed hcp-bcc phase region ${ }^{12}$ (Supplementary Fig. 6) which might result in an increase of the thermal conductivity. However, based on the $300 \mathrm{~K}$ data, there is no abrupt sizable change in the thermal conductivity of $\mathrm{Fe}_{0.85} \mathrm{Si}_{0.15}$ near $40 \mathrm{GPa}$ (where this transition is expected to occur at $300 \mathrm{~K}^{12}$ ); only a change in the

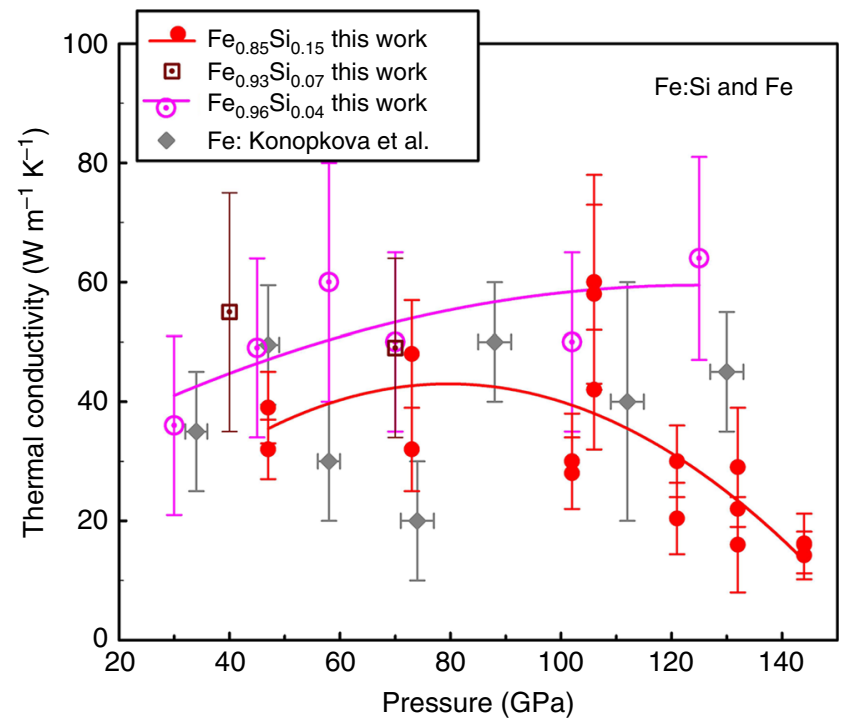

Fig. 2 High-pressure thermal conductivity of Fe-Si alloys at

2050-3300 K. Red and magenta curves are guides to the eye. Literature data for Fe at comparable high P-T conditions from ref. ${ }^{20}$ are plotted for comparison. Our results are representative of measurements with different laser powers, each corresponding to an averaging of usually 100 laser heating events using a streak camera 20,32 . The measurement uncertainties are typically $\approx 15-30 \%$. Effects of temperature on the thermal conductivity of $\mathrm{Fe}_{0.85} \mathrm{Si}_{0.15}$ alloy are shown in Supplementary Fig. 4. Pressure-temperature conditions for each measurement are listed in Supplementary Tables 1-3.

pressure slope is observed so the thermal conductivity remains approximately constant (Fig. 1). We thus conclude that the observed conductivity behavior for $\mathrm{Fe}_{0.85} \mathrm{Si}_{0.15}$ is mainly due to $\mathrm{Si}$ alloying effect in the hcp phase, instead of the hcp-bcc mixture in the sample.

\section{Discussion}

Extrapolation of our room-temperature pure hcp-Fe thermal conductivity data to relevant high-temperature conditions confirms the consistency of the TDTR results with the TH results. The TDTR data for pure hcp-Fe at the outermost core pressures and $300 \mathrm{~K}$ is about $120 \mathrm{~W} \mathrm{~m}^{-1} \mathrm{~K}^{-1}$ (Fig. 1). If we assume the temperature dependence of the pure hcp-Fe follows a $T^{-1 / 2}$ dependence as estimated by Konôpková et al. ${ }^{20}$, the thermal conductivity of hcp-Fe at the outermost core conditions $(\sim P=136 \mathrm{GPa}, T=3800-4800 \mathrm{~K})$ is estimated to be about $30-33.7 \mathrm{~W} \mathrm{~m}^{-1} \mathrm{~K}^{-1}$, nearly the same as that $\left(33 \pm 7 \mathrm{~W} \mathrm{~m}^{-1} \mathrm{~K}^{-1}\right)$ obtained by TH measurements (ref. ${ }^{20}$ and Supplementary Fig. 1). As for the Fe-Si alloys, however, the exact temperature dependence of thermal conductivity at high pressures likely varies with Si content and applied pressure (Fig. 2 and Supplementary Fig. 4), which remains relatively uncertain. Thus, it would be difficult to make unambiguous extrapolation of room-temperature TDTR data to high-temperature conditions and compare them with the high-temperature TH data. Nevertheless, we note that qualitatively these two sets of data correspond reasonably well as both sets of data demonstrate pressure dependencies with a broad maximum for $\mathrm{Fe}-\mathrm{Si}$ alloys (after about $40 \mathrm{GPa}$ at $300 \mathrm{~K}$ and around $80-100 \mathrm{GPa}$ at $2050-3300 \mathrm{~K})$. Moreover, given the $\mathrm{Si}$ alloying effect, it is expected that the $\mathrm{Fe}-\mathrm{Si}$ alloys would have weaker temperature dependences than the pure hcp-Fe, since the presence of impurities will enhance the scattering of carries (phonons and electrons) during the transport of energy. 
This qualitative behavior is clearly indicated in Supplementary Figs. 1-3.

We further compare our results with previous studies to disentangle the Si light element effect from the temperature-dependent thermal conductivity of $\mathrm{Fe}$ and $\mathrm{Fe}-\mathrm{Si}$ alloys at Earth's core pressures. The thermal conductivity of metals is mainly determined by the electronic contribution, which is the case for pure $\mathrm{Fe}$, where the lattice thermal conductivity is negligible ${ }^{24}$. However, at high pressure and room temperature, the electrical conductivities of hcp- $\mathrm{Fe}_{0.96} \mathrm{Si}_{0.04}$ and $\mathrm{Fe}_{0.84} \mathrm{Si}_{0.16}(\approx 2$ and $9 \mathrm{wt} \% \mathrm{Si}$, respectively) alloys, similar in composition to our samples, were found to be smaller than that of pure hcp-Fe by a factor of about 4 and $10^{22,27}$, respectively, due to the impurity effect. Compared with the intrinsic electron-phonon scattering, the impurity scattering effect plays a predominant role in influencing the thermal energy transport in $\mathrm{Fe}-\mathrm{Si}$ alloys at high $P-T$ conditions. Thus, the different temperature dependence of thermal conductivity among pure hcp-Fe, $\mathrm{Fe}_{0.96} \mathrm{Si}_{0.04}$, and $\mathrm{Fe}_{0.85} \mathrm{Si}_{0.15}$ could be explained by the $T$ dependence of the impurity scattering, as doping of silicon impurity likely flattens the temperature dependence of thermal conductivity. On the other hand, the variation in high $P-T$ thermal conductivity of $\mathrm{Fe}-\mathrm{Si}$ alloys is likely due to the $P-T$ effects on electron-impurity scattering contribution to the conductivity (see Supplementary Fig. 4 and Supplementary Note 1). We also note that because of a decrease in the electronic thermal conductivity contribution in $\mathrm{Fe}-\mathrm{Si}$ alloys, the phonon contribution (via, e.g., the electron-phonon and phononimpurity scatterings) to their total thermal conductivity is expected to play a non-negligible role for thermal transport ${ }^{34}$. The aforementioned dissimilarity in the $P$ - $T$-dependent thermal conductivity makes the conductivity values of hcp-Fe and hcp$\mathrm{Fe}_{0.96} \mathrm{Si}_{0.04}$ comparable with each other and about twice larger than hcp- $\mathrm{Fe}_{0.85} \mathrm{Si}_{0.15}$ at $P-T$ conditions relevant to Earth's outer core.

Prior studies reported that the electrical resistivity of solid $\mathrm{Fe}_{0.84} \mathrm{Si}_{0.16}$ at $\sim 136 \mathrm{GPa}$ and $3750 \mathrm{~K}$, i.e., outermost core conditions, is on the order of $\sim 1 \times 10^{-6} \Omega \mathrm{m}^{22,27}$. Using the WF law with the ideal Lorenz number, the corresponding thermal conductivity was estimated to be about $40-60$ and $90 \mathrm{~W} \mathrm{~m}^{-1} \mathrm{~K}^{-127}$, respectively. If we assume $\mathrm{Si}$ is the major light element with $\approx 15$ at $\%(\approx 8 \mathrm{wt} \%)$ in the outer core, these high literature values of inferred thermal conductivity of solid $\mathrm{Fe}_{0.84} \mathrm{Si}_{0.16}$ at outermost core conditions are much larger than the $\approx 20 \mathrm{~W} \mathrm{~m}^{-1} \mathrm{~K}^{-1}$ value for solid $\mathrm{Fe}_{0.85} \mathrm{Si}_{0.15}$ obtained by our direct measurements (See Table 1 for a summary of recent results on the electrical resistivity and thermal conductivity of $\mathrm{Fe}$ and $\mathrm{Fe}-\mathrm{Si}$ alloys at outer core conditions.). The large discrepancy may arise from the previously modeled temperature dependence of electrical resistivity at high pressure, or from using an assumed ideal Lorenz number at high $P-T$ conditions. We note that our direct thermal conductivity measurements do not involve these assumptions, yielding the robust conclusions concerning thermal evolution scenarios of the core (see geodynamic modeling below).

Our results on the thermal conductivity of solid $\mathrm{Fe}_{0.85} \mathrm{Si}_{0.15}$ at outer core $P-T$ conditions is expected to set an upper bound for that of the liquid outer core, as the extrapolation of our results (Supplementary Fig. 4 ) to the core temperatures $(>4000 \mathrm{~K}$ ) would not change it much, while the thermal conductivity of a material in molten phase that lacks crystallinity for heat conduction is typically smaller than in solid phase. For $\mathrm{Fe}$ and Fe-light element alloys, the effect of melting is expected to reduce the thermal conductivity of the solid phase by $\approx 20 \%$ or less ${ }^{15,18,20-22,34-37}$. For instance, Silber et al. ${ }^{36}$ recently reported that at pressures from 3 to $9 \mathrm{GPa}$ the electrical resistivity (inversely proportional to the electronic thermal conductivity using WF law) of Fe alloyed with $4.5 \mathrm{wt} \%$ Si abruptly increases by $\sim 10^{-7} \Omega \mathrm{m}(\sim 10 \%)$ or less

\section{Table 1 Recent experimental and computational results of electrical resistivity $\rho$ and thermal conductivity $\Lambda$ of Fe and Fe-Si alloys at outer core conditions.}

\begin{tabular}{|c|c|c|c|c|}
\hline Composition & $\begin{array}{l}\rho(\mu \Omega \\
\mathrm{cm})\end{array}$ & $\begin{array}{l}\Lambda\left(W m^{-1}\right. \\
\left.K^{-1}\right)\end{array}$ & Method & Reference \\
\hline hcp Fe & $\sim 90$ & $\sim 100$ & C & 15 \\
\hline hсp Fe & NA & $\sim 33$ & DTCM & 20 \\
\hline hсp $\mathrm{Fe}$ & $\sim 40$ & $\sim 226^{a}$ & ERM & 21 \\
\hline hсp $\mathrm{Fe}$ & $\sim 60-130$ & $\sim 67-145^{a}$ & ERM & 22 \\
\hline Liquid Fe & $\sim 70$ & $\sim 140$ & $\mathrm{C}$ & 16 \\
\hline Liquid Fe & $\sim 70$ & $\sim 130$ & C & 17 \\
\hline hсp $\mathrm{Fe}_{0.85} \mathrm{Si}_{0.15}$ & NA & $\sim 20$ & DTCM & This study \\
\hline hcp $\mathrm{Fe}_{0.84} \mathrm{Si}_{0.16}$ & 150-215 & $\sim 41-60^{a}$ & ERM & 22 \\
\hline hcp $\mathrm{Fe}_{0.78} \mathrm{Si}_{0.22}$ & $\sim 100$ & $\sim 90^{a}$ & ERM & 25 \\
\hline Liquid $\mathrm{Fe}_{0.875} \mathrm{Si}_{0.125}$ & $\sim 90$ & $\sim 110$ & C & 17 \\
\hline hcp $\mathrm{Fe}_{0.65} \mathrm{Ni}_{0.1} \mathrm{Si}_{0.25}$ & $\sim 112$ & $\sim 87^{a}$ & $\mathrm{ERM}+\mathrm{C}$ & 27 \\
\hline
\end{tabular}

Method: $C$ calculation, DTCM direct thermal conductivity measurement, ERM electrical resistivity measurement.

NA not applicable.

aThermal conductivity was inferred from electrical resistivity via WF law.

Table 2 Recent results on the change in electrical resistivity $\Delta \rho$ of Fe and Fe-Si alloys upon melting.

\begin{tabular}{lllll} 
Composition & $\boldsymbol{P}(\mathbf{G P a})$ & $\boldsymbol{\Delta} \boldsymbol{\rho}(\boldsymbol{\mu} \mathbf{\Omega} \mathbf{~ c m})$ & Method & Reference \\
\hline $\mathrm{Fe}$ & 329 & $\sim+10$ & $\mathrm{C}$ & 18 \\
$\mathrm{Fe}$ & 51 & $\sim+20-30$ & ERM & 21 \\
$\mathrm{Fe}$ & 12 & $\sim+10$ & ERM & 35 \\
$\mathrm{Fe}$ & 5 & $\sim+20$ & ERM & 37 \\
$\mathrm{Fe}_{0.91} \mathrm{Si}_{0.09}$ & 9 & $\sim+5$ & ERM & 36 \\
$\mathrm{Fe}_{0.82} \mathrm{Si}_{0.18}$ & 10 & $\sim-50$ & ERM & 38 \\
$\mathrm{Fe}_{0.82} \mathrm{Si}_{0.1} \mathrm{O}_{0.08}$ & 329 & $\sim+15$ & $\mathrm{C}$ & 18 \\
\hline
\end{tabular}

Method: $C$ calculation, ERM electrical resistivity measurement.

as it undergoes a solid-to-liquid transition, and such increase in resistivity is expected to be also present at higher pressures ${ }^{18}$. We note, however, that recent electrical resistivity data for $\mathrm{Fe}-\mathrm{Si}$ alloys by Pommier et al. ${ }^{38}$ show an opposite trend, at odds with most literature results where the electrical resistivity of metals and their alloys typically increases with temperature and upon melting $15,18,21,25,34-37$ (see Table 2 for a summary of recent results on the change in electrical resistivity of $\mathrm{Fe}$ and $\mathrm{Fe}-\mathrm{Si}$ alloys upon melting).

Assuming an outer core with 15 at\% Si being the major light element, the significant reduction of Fe thermal conductivity by about twofolds caused by alloying 15 at\% $\mathrm{Si}$ at outer core conditions, as indicated by our data, provides crucial constraints on the thermal state and geodynamo of the outer core as well as the age of the inner core. Our result for the low thermal conductivity of $\mathrm{Fe}_{0.85} \mathrm{Si}_{0.15}$ alloy at outer core conditions, $\approx 20 \mathrm{~W} \mathrm{~m}^{-1} \mathrm{~K}^{-1}$, represents the first direct measurement that pins down the outer core thermal conductivity to a low-end value estimated in ref. ${ }^{20}$. It further considerably lowers the power requirements of a thermally driven geodynamo compared with recently proposed scenarios, which in turn requires lower initial core temperatures and consequently a potentially older inner core ${ }^{2,10}$. More specifically, the core's thermal conductivity provides a lower bound on the power that needs to be extracted from the core at the CMB to drive a thermal geodynamo. The thermal geodynamo can obviously operate with higher power, and the real value of this power is imposed by the CMB heat flow, which is itself controlled by mantle convection and is estimated to be in the range of 5-17 $\mathrm{TW}^{39}$. The 
a
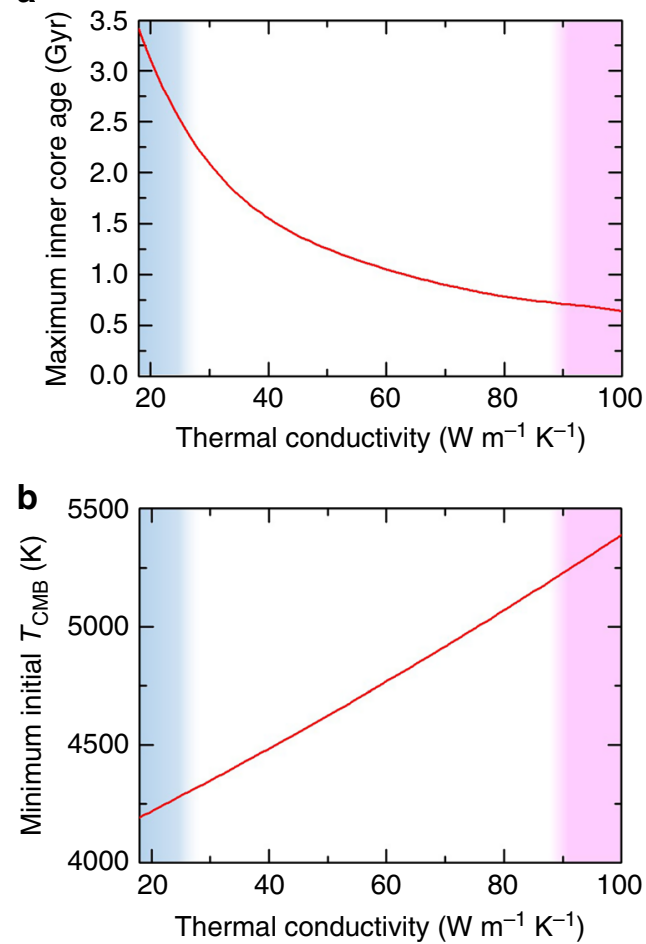

Fig. 3 Effects of core thermal conductivity on its thermal evolution.

a maximum inner core age and $\mathbf{b}$ minimum initial core-mantle boundary (CMB) temperature as a function of thermal conductivity of the core.

Results are obtained with a thermal evolution model assuming an isentropic $\mathrm{CMB}$ heat flow at each time, which is the minimum to maintain a magnetic field by thermal convection alone. Blue shaded areas represent the range of $\mathrm{Fe}_{0.85} \mathrm{Si}_{0.15}$ thermal conductivity at outer core $P-T$ conditions indicated by our study, while red shaded areas represent the high thermal conductivity values of $\mathrm{Fe}-\mathrm{Si}$ alloys inferred from literature results, see e.g., refs. ${ }^{25,27}$.

thermal convection lower bound is defined by the critical CMB heat flow at which convection turns on, i.e., the isentropic heat flow, given by the product of thermal conductivity and isentropic temperature gradient. The actual heat flow can be lower thanc the isentropic value if the compositional convection occurs, owing to the inner core growth ${ }^{5}$ or light element extraction ${ }^{6-9}$, which is a tenet of buoyancy-driven dynamos ${ }^{7,40}$. For a thermal conductivity of $\approx 20 \mathrm{~W} \mathrm{~m}^{-1} \mathrm{~K}^{-1}$, the minimum heat flow is $\approx 3 \mathrm{TW}^{41}$, i.e., smaller than the lower bound of an estimated modern CMB heat flow $^{39}$, while for conductivities larger than $\approx 115 \mathrm{~W} \mathrm{~m}^{-1} \mathrm{~K}^{-1}$, the minimum heat flow is larger than the upper estimate of a modern $\mathrm{CMB}$ heat flow. To illustrate the key role played by the thermal conductivity on the core evolution, we further calculated the maximum inner core age and minimum initial CMB temperature for a wide range of core thermal conductivity (Fig. 3). For simplicity, we consider that the dynamo before the inner core nucleation runs on heat alone, i.e., no other source than thermal is available at that time. We computed the thermal evolution of the core for a $\mathrm{CMB}$ heat flow always equal to the isentropic value, i.e., the minimum requirement to initiate thermal convection and run a dynamo. The high-end values $\left(>90 \mathrm{~W} \mathrm{~m}^{-1} \mathrm{~K}^{-1}\right.$, red shaded areas in Fig. 3) obtained by previous theoretical predictions ${ }^{16,17}$ and electrical resistivity measurements ${ }^{25,27}$ combined with calculations using the WF law with ideal Lorenz number result in unrealistically high CMB temperature in the early Earth ${ }^{10}$. By contrast, the value obtained by our direct thermal conductivity measurements $\left(\approx 20 \mathrm{~W} \mathrm{~m}{ }^{-1} \mathrm{~K}^{-1}\right.$, blue shaded areas in Fig. 3$)$ leads to reasonable bounds on thermal evolution scenarios.
Our results further indicate that Earth's dynamo could have been running on the thermal energy alone throughout its history with the additional help from compositional buoyancy when the inner core started to crystallize. The low thermal conductivity $\left(\approx 20 \mathrm{~W} \mathrm{~m}^{-1} \mathrm{~K}^{-1}\right)$ of the outer core enables a purely thermally driven dynamo with an initial CMB temperature on the order of $4500 \mathrm{~K}$, which is a geochemically acceptable value ${ }^{42}$ from the standpoint of core formation. The significant reduction of Fe thermal conductivity due to the Si impurity effect could be general for other candidate light elements in the core. Additional direct high $P-T$ thermal conductivity measurements on $\mathrm{O}-, \mathrm{S}-$, and $\mathrm{C}-$ bearing binaries and more realistic ternary $\mathrm{Fe}-$ light element systems are required to precisely quantify the role played by these elements. These future studies could strengthen the conclusion that, due to the low core thermal conductivity, the geodynamo can run on heat alone for the entire age of the Earth without the help of compositional convection.

\begin{abstract}
Methods
Starting materials and sample preparation. Single crystals of pure Fe for TDTR experiments at $300 \mathrm{~K}$ were synthesized by Princeton Scientific Corporation, Princeton, NJ. At ambient conditions, the pure Fe is in bcc phase with (100) orientation. Powder samples of pure $\mathrm{Fe}$ and chemically homogeneous $\mathrm{Fe}_{1-\mathrm{x}} \mathrm{Si}_{\mathrm{x}}(x=0.04$ and 0.15 ) alloys were from Goodfellow Corporation, and their crystal structures were also in bcc phase characterized by X-ray diffraction. The chemical composition of each alloy was confirmed to be $\mathrm{Fe}_{0.96} \mathrm{Si}_{0.04}$ and $\mathrm{Fe}_{0.85} \mathrm{Si}_{0.15}$ by an electron microprobe ${ }^{12}$. Before being loaded into the high-pressure DAC, the single-crystal samples were cut to $\approx 50 \times 50 \mu \mathrm{m}^{2}$ and a thickness of $\approx 30 \mu \mathrm{m}$ using focused ion beam (FIB) in Center for High Pressure Science and Technology Advanced Research (HPSTAR), Shanghai, and powder samples were pressed to a disk shape with a diameter of $\approx 50 \mu \mathrm{m}$ and a thickness of $\approx 10 \mu \mathrm{m}$.

The $\mathrm{Fe}_{0.85} \mathrm{Si}_{0.15}$ alloy was synthesized in an end-loaded 150 -ton piston-cylinder press at Institut de physique du globe de Paris, by equilibrating molten metal with molten silicate at fixed temperature and oxygen fugacity. For this, natural fresh MORB from the mid-Atlantic ridge (GRA-N16-6) was ground and mixed with Fe and FeSi, then fully melted and equilibrated at $2 \mathrm{GPa}$ and $1800^{\circ} \mathrm{C}$ for $120 \mathrm{~s}$, using an $\mathrm{MgO}$ capsule, a graphite furnace and $\mathrm{BaCO}_{3}$ cell. After quench, the metal had fully coalesced to a spherical ball surrounded by silicate glass; its homogeneity and composition were analyzed by a scanning electron microscope, and it was then crushed for loading in DAC experiments. All the samples for high-temperature TH measurements are polycrystalline.

To measure the thermal conductivity at high pressures and $300 \mathrm{~K}$, the samples were then coated with $\approx 80$-nm thick Al film and loaded, together with a ruby ball, into a symmetric DAC with a culet size of 200 or $300 \mu \mathrm{m}$ and a Re gasket. Silicone oil (CAS No. 63148-62-9 from ACROS ORGANICS) was used as the pressure medium. The pressure was determined by fluorescence spectrum of the ruby ${ }^{43}$ with a typical uncertainty of less than $5 \%$.

In TH experiments to high temperature, the samples thinned down (by squeezing between two diamonds) to $\sim 4 \mu \mathrm{m}$ were loaded in a symmetric DAC using $\mathrm{KCl}$ as a pressure medium and thermal insulator. The sample position and thickness and the distances between the sample surface and diamond tips were measured at high pressure using optical spectroscopy of the interference fringes recorded in the reflectivity spectra from the cavity without the sample and from the sample surfaces from both sides ${ }^{20,32}$. The refractive index of $\mathrm{KCl}$ was determined by extrapolating linearly the results as a function of density in ref. ${ }^{44}$ to higher pressures. Pressure was determined from the position of the Raman edge of the stressed diamond anvil tip ${ }^{45}$.
\end{abstract}

Thermal conductivity at high pressure and room temperature. Thermal conductivities of $\mathrm{Fe}$ and $\mathrm{Fe}-\mathrm{Si}$ alloys at high pressure and room temperature were measured using an ultrafast optical pump-probe method, TDTR. In our TDTR measurements, the output of a Ti:sapphire oscillator laser was split into pump and probe beams. The pump beam heated up the $\mathrm{Al}$ film coated on the sample and created temperature variations. The resulting optical reflectivity change of the $\mathrm{Al}$ film as a function of time was measured by the probe beam that was delayed by passing through a mechanical stage. The in-phase $V_{\text {in }}$ (real part) and out-of-phase $V_{\text {out }}$ (imaginary part) components of the variations of the reflected probe beam intensity, synchronous with the $8.7 \mathrm{MHz}$ modulation frequency of the pump beam, were detected by a Si fast photodiode and an RF lock-in amplifier. Detailed descriptions of the TDTR method are discussed elsewhere, see, for example, refs. 46,47 .

To determine the thermal conductivity of the sample, we compared the ratio $-V_{\text {in }} / V_{\text {out }}$ as a function of delayed time between pump and probe beams to thermal model calculations that take into account heat flow into the sample and into the pressure medium silicone oil ${ }^{48,49}$. Example data for hcp Fe at high pressures along 
with calculations by the thermal model are shown in Supplementary Fig. 7. There are several parameters in our thermal model, including laser spot size, thickness of $\mathrm{Al} \mathrm{film}$, thermal conductivity, and heat capacity of each layer, but the thermal conductivity of the sample is the only significant unknown and free parameter to be determined. Under our experimental geometry and conditions, the ratio $-V_{\text {in }} / V_{\text {out }}$ during the delay time of few hundred picoseconds is most sensitive to and scales with sum of the thermal effusivity of the sample and silicone oil divided by the heat capacity per unit area of the $\mathrm{Al} \mathrm{film}$, see ref. ${ }^{50}$ for details. The $\mathrm{Al}$ thickness at ambient pressure was measured in situ by picosecond acoustics; we estimated the changes in $\mathrm{Al}$ thickness at high pressures following a method developed in ref. ${ }^{51}$ : Al thickness decreases by $7.8 \%$ at $25 \mathrm{GPa}$, by $10.3 \%$ at $40 \mathrm{GPa}$, by $13.1 \%$ at $70 \mathrm{GPa}$, and $15.4 \%$ at $120 \mathrm{GPa}$. In addition, at the modulation frequency of the pump beam (8.7 MHz), the thermal penetration depths in Fe, Fe-Si alloy, and silicone oil are of the order of hundreds of nanometers ${ }^{52}$, and therefore our thermal model calculations are insensitive to their thicknesses $(\sim 10 \mu \mathrm{m})$, see Supplementary Fig. 8a, b. Since the Al thermal conductivity at ambient pressure is large $\left(\approx 200 \mathrm{~W} \mathrm{~m}^{-1} \mathrm{~K}^{-1}\right)^{50}$ and has minimal effects on the thermal model calculations (Supplementary Fig. 8c), we fixed this value at high pressures. We estimated the $\mathrm{Al}$ heat capacity at high pressures from literature data for the atomic density and elastic constants at high pressures along with calculations of Debye temperature, see ref. ${ }^{52}$ for details. The pressure dependent thermal effusivity, square root of the product of thermal conductivity and volumetric heat capacity, of silicone oil to $24 \mathrm{GPa}$ was taken from ref. ${ }^{33}$; the thermal effusivity from 24 to $120 \mathrm{GPa}$ was estimated by extrapolation of the lower pressure data that were fitted into a polynomial, assuming the silicone oil remains in an amorphous phase at these pressures. Note that the thermal effusivity of silicone oil at high pressures is much smaller than that of the $\mathrm{Fe}$ and $\mathrm{Fe}-\mathrm{Si}$ alloys, which significantly reduces the uncertainty of the measured thermal conductivity of the sample; the exceptionally low thermal effusivity of silicone oil has minor influences on the thermal model calculations, typically less than $5 \%$ uncertainty, see Supplementary Fig. 8d.

The volumetric heat capacity of the bcc Fe at ambient pressure and room temperature is $3.54 \mathrm{~J} \mathrm{~cm}^{-3} \mathrm{~K}^{-1}$, and its pressure dependence is taken from the results of ref. ${ }^{54}$ along with the equation of state (EOS) from ref. ${ }^{55}$, where the relatively small electronic contribution to the heat capacity is further reduced at high pressures. For the hcp Fe, the lattice contribution to the heat capacity was taken from the results by Murphy et al. ${ }^{56}$. Though its electronic contribution is not well known, Wasserman et al. ${ }^{57}$ showed that, for $f c c \mathrm{Fe}$, the electronic contribution to the heat capacity is much smaller than the lattice contribution, in particular at room temperature and higher pressures. We assumed the hcp Fe has similar property as suggested by ref. 58 and thus its lattice heat capacity is predominantly and reasonably represents the total heat capacity of hcp Fe at room temperature and high pressures.

On the other hand, the volumetric heat capacities of the $\mathrm{Fe}_{0.96} \mathrm{Si}_{0.04}$ and $\mathrm{Fe}_{0.85} \mathrm{Si}_{0.15}$ alloys at room temperature and high pressures are not known. We first estimated their heat capacities at ambient conditions to be 3.72 and $4.22 \mathrm{~J} \mathrm{~cm}^{-3} \mathrm{~K}^{-1}$, respectively, by interpolating the ambient heat capacities between pure bcc Fe and $\mathrm{FeSi}^{59}$ for 4 and 15 at\% of Si. We then assumed both the $\mathrm{Fe}_{0.96} \mathrm{Si}_{0.04}$ and $\mathrm{Fe}_{0.85} \mathrm{Si}_{0.15}$ have a similar pressure dependence to that of the FeSi as calculated in ref. 59 . Since the electrical resistivities of $\mathrm{Fe}_{0.96} \mathrm{Si}_{0.04}$ and $\mathrm{Fe}_{0.85} \mathrm{Si}_{0.15}$ are larger than $\mathrm{Fe}$, their total heat capacity is predominantly determined by the lattice contribution. Finally, by evaluating the sensitivity of the thermal model to input parameters, we calculated the uncertainty in the thermal conductivity of $\mathrm{Fe}$ and $\mathrm{Fe}-\mathrm{Si}$ alloys resulting from the uncertainty in each of the parameters used in our thermal model (see, for example, refs. ${ }^{50,60}$ for details of the uncertainty evaluation, and example tests in Supplementary Fig. 8). Importantly, precise determination of the $\mathrm{Fe}$ and $\mathrm{Fe}-\mathrm{Si}$ alloys thermal conductivity requires the model to have higher sensitivity to their thermal conductivity but lower sensitivity to other input parameters. We found that uncertainties in all the parameters propagate to $\approx 10 \%$ error in the measured thermal conductivity before $30 \mathrm{GPa}, \approx 20 \%$ error at $60 \mathrm{GPa}$, and $\approx 25 \%$ error at $120 \mathrm{GPa}$.

Thermal conductivity at high pressure and temperature. Thermal conductivity at high pressure and high temperature was measured by the $\mathrm{TH}$ technique similar to those reported in refs. ${ }^{20,32}$. In our experiments, the bulk of a several $\mu \mathrm{m}$ thick sample preheated by continuous-wave lasers from both sides is probed by launching a thermal wave created by sending a microsecond ( $\mu \mathrm{s})$ long pulse from one sample side and recording its temperature history via a time resolved spectroradiometry from both samples sides (Supplementary Fig. 9). These temperature evolutions were approximated by two-dimensional (axially symmetric) FE model calculations using the experimentally determined sample geometry ${ }^{20,32,61}$. The EOS of $\mathrm{Fe}_{0.85} \mathrm{Si}_{0.15}$ is from ref. ${ }^{12}$, and the $\mathrm{EOS}$ of $\mathrm{KCl}$ is from ref. ${ }^{62}$. The two major parameters to fit the data are the thermal conductivity of the sample and the medium $(\mathrm{KCl})$. The error bars are estimated as combined uncertainties of fitting, input material and geometrical parameters, and other assumptions (e.g., neglecting thermal expansion).

\section{Data availability}

All data supporting the findings of this study are available within the paper or available from the corresponding authors upon request.
Received: 18 November 2019; Accepted: 4 June 2020;

Published online: 03 July 2020

\section{References}

1. Hirose, K., Labrosse, S. \& Hernlund, J. Composition and State of the Core. Annu. Rev. Earth Planet. Sci. 41, 657-691 (2013).

2. Labrosse, S. Thermal and magnetic evolution of the Earth's core. Phys. Earth Planet. Inter 140, 127-143 (2003).

3. Buffett, B. A. The thermal state of Earth's Core. Science 299, 1675-1677 (2003).

4. Lay, T., Hernlund, J. \& Buffett, B. Core-mantle boundary heat flow. Nat. Geosci. 1, 25-32 (2008).

5. Loper, D. E. The gravitationally powered dynamo. Geophys. J. R. astr. Soc. 54, 389-404 (1978)

6. Hirose, K. et al. Crystallization of silicon dioxide and compositional evolution of the Earth's core. Nature 543, 99-102 (2017).

7. Badro, J., Siebert, J. \& Nimmo, F. An early geodynamo driven by exsolution of mantle components from Earth's core. Nature 536, 326-328 (2016)

8. O'Rourke, J. G. \& Stevenson, D. J. Powering Earth's dynamo with magnesium precipitation from the core. Nature 529, 387-389 (2016).

9. Buffett, B. A., Garnero, E. J. \& Jeanloz, R. Sediments at the top of Earth's Core. Science 290, 1338-1342 (2000).

10. Labrosse, S. Thermal evolution of the core with a high thermal conductivity. Phys. Earth Planet. Inter 247, 36-55 (2015).

11. Olson, P. The new core paradox. Science 342, 431 (2013).

12. Lin, J. F. et al. Phase relations of Fe-Si alloy in Earth's core. Geophys. Res. Lett. 36, L06306 (2009)

13. Li, J. \& Fei, Y. Experimental constraints on core composition. Treatise Geochem. 2, 568 (2003).

14. Badro, J. et al. Effect of light elements on the sound velocities in solid iron: implications for the composition of Earth's core. Earth Planet. Sci. Lett. 254 233-238 (2007)

15. $\mathrm{Xu}, \mathrm{J}$. et al. Thermal conductivity and electrical resistivity of solid iron at Earth's core conditions from first principles. Phys. Rev. Lett. 121, 096601 (2018).

16. Pozzo, M., Davies, C., Gubbins, D. \& Alfe, D. Thermal and electrical conductivity of iron at Earth's core conditions. Nature 485, 355 (2012).

17. deKoker, N., Steinle-Neumann, G. \& Vlček, V. Electrical resistivity and thermal conductivity of liquid Fe alloys at high $\mathrm{P}$ and $\mathrm{T}$, and heat flux in Earth's core. Proc. Natl Acad. Sci. USA 109, 4070 (2012).

18. Pozzo, M., Davies, C., Gubbins, D. \& Alfè, D. Thermal and electrical conductivity of solid iron and iron-silicon mixtures at Earth's core conditions. Earth Planet. Sci. Lett. 393, 159-164 (2014).

19. Tarduno, J., Cottrell, R., Davis, W., Nimmo, F. \& Bono, R. A hadean to paleoarchean geodynamo recored by single zircon crystals. Science 349, 521 (2015).

20. Konopkova, Z., McWilliams, R. S., Gomez-Perez, N. \& Goncharov, A. F. Direct measurement of thermal conductivity in solid iron at planetary core conditions. Nature 534, 99-101 (2016).

21. Ohta, K., Kuwayama, Y., Hirose, K., Shimizu, K. \& Ohishi, Y. Experimental determination of the electrical resistivity of iron at Earth's core conditions. Nature 534, 95-98 (2016)

22. Seagle, C. T., Cottrell, E., Fei, Y., Hummer, D. R. \& Prakapenka, V. B. Electrical and thermal transport properties of iron and iron-silicon alloy at high pressure. Geophys. Res. Lett. 40, 5377-5381 (2013).

23. Stacey, F. D. \& Loper, D. E. A revised estimate of the conductivity of iron alloy at high pressure and implications for the core energy balance. Phys. Earth Planet. Inter 161, 13-18 (2007).

24. Stacey, F. D. \& Anderson, O. L. Electrical and thermal conductivities of Fe-Ni-Si alloy under core conditions. Phys. Earth Planet. Inter 124, 153-162 (2001).

25. Gomi, H. et al. The high conductivity of iron and thermal evolution of the Earth's core. Phys. Earth Planet. Inter 224, 88-103 (2013).

26. Gomi, H. \& Hirose, K. Electrical resistivity and thermal conductivity of hcp $\mathrm{Fe}-\mathrm{Ni}$ alloys under high pressure: Implications for thermal convection in the Earth's core. Phys. Earth Planet. Inter 247, 2-10 (2015).

27. Gomi, H., Hirose, K., Akai, H. \& Fei, Y. Electrical resistivity of substitutionally disordered hcp Fe-Si and Fe-Ni alloys: chemically-induced resistivity saturation in the Earth's core. Earth Planet. Sci. Lett. 451, 51-61 (2016).

28. Hsieh, W.-P., Deschamps, F., Okuchi, T. \& Lin, J.-F. Reduced lattice thermal conductivity of Fe-bearing bridgmanite in Earth's deep mantle. J. Geophys. Res. Solid Earth 122, 4900-4917 (2017).

29. Hsieh, W.-P., Deschamps, F., Okuchi, T. \& Lin, J.-F. Effects of iron on the lattice thermal conductivity of Earth's deep mantle and implications for mantle dynamics. Proc. Natl Acad. Sci. USA 115, 4099-4104 (2018). 
30. Pourovskii, L. V., Mravlje, J., Ferrero, M., Parcollet, O. \& Abrikosov, I. A Impact of electronic correlations on the equation of state and transport in epsilon-Fe. Phys. Rev. B 90, 155120 (2014).

31. Glazyrin, K. et al. Importance of correlation effects in HCP iron revealed by a pressure-induced electronic topological transition. Phys. Rev. Lett. 110, 117206 (2013).

32. McWilliams, R. S., Konopkova, Z. \& Goncharov, A. F. A flash heating method for measuring thermal conductivity at high pressure and temperature: application to Pt. Phys. Earth Planet. Inter 247, 17-26 (2015).

33. Komabayashi, T. et al. Phase transition boundary between fcc and hcp structures in $\mathrm{Fe}-\mathrm{Si}$ alloy and its implications for terrestrial planetary cores. Am. Miner. 104, 94-99 (2019).

34. Secco, R. A. Thermal conductivity and Seebeck coefficient of Fe and Fe-Si alloys: implications for variable Lorenz number. Phys. Earth Planet. Inter 265, 23-34 (2017).

35. Silber, R. E., Secco, R. A., Yong, W. \& Littleton, J. A. H. Electrical resistivity of liquid $\mathrm{Fe}$ to $12 \mathrm{GPa}$ : Implications for heat flow in cores of terrestrial bodies. Sci. Rep. 8, 10758 (2018).

36. Silber, R. E., Secco, R. A., Yong, W. \& Littleton, J. A. H. Heat flow in Earth's core from invariant electrical resistivity of $\mathrm{Fe}-\mathrm{Si}$ on the melting boundary to 9 GPa: do light elements matter? J. Geophys. Res. Solid Earth 124, 5521-5543 (2019).

37. Deng, L., Seagle, C., Fei, Y. \& Shahar, A. High pressure and temperature electrical resistivity of iron and implications for planetary cores. Geophys. Res. Lett. 40, 33-37 (2013).

38. Pommier, A., Leinenweber, K. \& Tran, T. Mercury's thermal evolution controlled by an insulating liquid outermost core? Earth Planet. Sci. Lett. 517, 125-134 (2019).

39. Jaupart, C., Labrosse, S., Lucazeau, F. \& Mareschal, J.-C. Temperature, heat, and energy in the mantle of the Earth. Treatise Geophys. 2nd Ed. 7, 218-251 (2015).

40. O'Rourke, J. G., Korenaga, J. \& Stevenson, D. J. Thermal evolution of Earth with magnesium precipitation in the core. Earth Planet. Sci. Lett. 458, 263-272 (2017).

41. Labrosse, S. Thermal state and evolution of the Earth core and deep mantle. Deep Earth Phys. Chem. Low. mantle core, Geophys. Monogr. 217, 43-54 (2016).

42. Badro, J., Brodholt, J. P., Piet, H., Siebert, J. \& Ryerson, F. J. Core formation and core composition from coupled geochemical and geophysical constraints. Proc. Natl Acad. Sci. USA. 112, 12310-12314 (2015).

43. Mao, H. K., Bell, P. M., Shaner, J. W. \& Steinberg, D. J. Specific volume measurements of $\mathrm{Cu}, \mathrm{Mo}, \mathrm{Pd}$, and $\mathrm{Ag}$ and calibration of the ruby $\mathrm{R} 1$ fluorescence pressure gauge from 0.06 to 1 Mbar. J. Appl. Phys. 49, 3276-3283 (1978).

44. Johannsen, P. G. et al. Refractive index of the alkali halides. II. Effect of pressure on the refractive index of 11 alkali halides. Phys. Rev. B 55, 6865-6870 (1997).

45. Akahama, Y. \& Kawamura, H. Pressure calibration of diamond anvil Raman gauge to $410 \mathrm{GPa}$. J. Phys. Conf. Ser. 215, 012195 (2010).

46. Kang, K., Koh, Y. K., Chiritescu, C., Zheng, X. \& Cahill, D. G. Two-tint pumpprobe measurements using a femtosecond laser oscillator and sharp-edged optical filters. Rev. Sci. Instrum. 79, 114901 (2008).

47. Cahill, D. G. Analysis of heat flow in layered structures for time-domain thermoreflectance. Rev. Sci. Instrum. 75, 5119-5122 (2004).

48. Ge, Z., Cahill, D. \& Braun, P. Thermal conductance of hydrophilic and hydrophobic interfaces. Phys. Rev. Lett. 96, 186101 (2006).

49. Schmidt, A., Chiesa, M., Chen, X. \& Chen, G. An optical pump-probe technique for measuring the thermal conductivity of liquids. Rev. Sci. Instrum. 79, 064902 (2008).

50. Zheng, X., Cahill, D. G., Krasnochtchekov, P., Averback, R. S. \& Zhao, J. C. High-throughput thermal conductivity measurements of nickel solid solutions and the applicability of the Wiedemann-Franz law. Acta Mater. 55, 5177-5185 (2007).

51. Chen, B., Hsieh, W.-P., Cahill, D. G., Trinkle, D. R. \& Li, J. Thermal conductivity of compressed $\mathrm{H} 2 \mathrm{O}$ to $22 \mathrm{GPa}$ : A test of the Leibfried-Schlömann equation. Phys. Rev. B 83, 132301 (2011)

52. Hsieh, W.-P., Chen, B., Li, J., Keblinski, P. \& Cahill, D. G. Pressure tuning of the thermal conductivity of the layered muscovite crystal. Phys. Rev. B 80, 180302 (2009).

53. Hsieh, W.-P. Thermal conductivity of methanol-ethanol mixture and silicone oil at high pressures. J. Appl. Phys. 117, 235901 (2015).

54. Sha, X. \& Cohen, R. E. Lattice dynamics and thermodynamics of bcc iron under pressure: first-principles linear response study. Phys. Rev. B 73, 104303 (2006).

55. Jephcoat, A. P., Mao, H. K. \& Bell, P. M. Static compression of iron to $78 \mathrm{GPa}$ with rare gas solids as pressure-transmitting media. J. Geophys. Res. 91, 4677-4684 (1986).

56. Murphy, C. A., Jackson, J. M., Sturhahn, W. \& Chen, B. Grüneisen parameter of hcp-Fe to $171 \mathrm{GPa}$. Geophys. Res. Lett. 38, L24306 (2011).
57. Wasserman, E., Stixrude, L. \& Cohen, R. E. Thermal properties of iron at high pressures and temperatures. Phys. Rev. B 53, 8296-8309 (1996).

58. Alfe, D., Price, G. D. \& Gillan, M. J. Thermodynamics of hexagonal-closepacked iron under Earth's core conditions. Phys. Rev. B 64, 045123 (2001).

59. Zhao, K., Jiang, G. \& Wang, L. Electronic and thermodynamic properties of B2-FeSi from first principles. Phys. B Phys. Condens. Matter 406, 363-367 (2011).

60. Cahill, D. G. \& Watanabe, F. Thermal conductivity of isotopically pure and Ge-doped Si epitaxial layers from 300 to 550 K. Phys. Rev. B 70, 235322 (2004).

61. Montoya, J. A. \& Goncharov, A. F. Finite element calculations of the time dependent thermal fluxes in the laser-heated diamond anvil cell. J. Appl. Phys. 111, 112617 (2012).

62. Dewaele, A. et al. High-pressure-high-temperature equation of state of $\mathrm{KCl}$ and KBr. Phys. Rev. B 85, 214105 (2012).

\section{Acknowledgements}

The work by W.P.H. was supported by the Academia Sinica and the Ministry of Science and Technology of Taiwan, Republic of China, under Contract AS-CDA-106-M02, 106-2116M-001-022, and 107-2628-M-001-004-MY3. W.P.H. acknowledges the fellowship from the Foundation for the Advancement of Outstanding Scholarship, Taiwan. The work at Carnegie was supported by the NSF (grant numbers DMR-1039807, EAR-1520648, EAR/IF1128867, EAR-1763287, EAR-1901813), the Army Research Office (grant number W911NF-19-2-0172), the Deep Carbon Observatory, the National Natural Science Foundation of China (grant number 21473211, 51672279, 11874361, and 11774354), the Chinese Academy of Science (grant number YZJJ2020QN22 and YZ201524) and the CASHIPS Director's Fund (Grant No. YZJJ201705). S.S.L. was partially supported through the Helmholtz Young Investigators Group CLEAR (VH-NG-1325). J.F.L. acknowledges support from the National Science Foundation (EAR-1901801) and the Deep Carbon Observatory The authors acknowledge Dr. James Badro for his help with the sample preparation, and Dr. Youjun Zhang for cutting single-crystal samples using a focused ion beam system in the Center for High Pressure Science and Technology Advanced Research.

\section{Author contributions}

W.P.H., A.F.G. and J.F.L. conceived and designed the project. W.P.H., A.F.G., N.H., S.S.L and I.C. conducted experiments and analyzed data. S.L. performed core evolution models. W.P.H., A.F.G., S.L., F. D. and J.F.L. wrote the manuscript. All authors reviewed and commented on the manuscript.

\section{Competing interests}

The authors declare no competing interests.

\section{Additional information}

Supplementary information is available for this paper at https://doi.org/10.1038/s41467 020-17106-7.

Correspondence and requests for materials should be addressed to W.-P.H., A.F.G. or J.-F.L

Peer review information Nature Communications thanks Eiji Ohtani and the other, anonymous, reviewer(s) for their contribution to the peer review of this work. Peer reviewer reports are available.

Reprints and permission information is available at http://www.nature.com/reprints

Publisher's note Springer Nature remains neutral with regard to jurisdictional claims in published maps and institutional affiliations.

Open Access This article is licensed under a Creative Commons Attribution 4.0 International License, which permits use, sharing, adaptation, distribution and reproduction in any medium or format, as long as you give appropriate credit to the original author(s) and the source, provide a link to the Creative Commons license, and indicate if changes were made. The images or other third party material in this article are included in the article's Creative Commons license, unless indicated otherwise in a credit line to the material. If material is not included in the article's Creative Commons license and your intended use is not permitted by statutory regulation or exceeds the permitted use, you will need to obtain permission directly from the copyright holder. To view a copy of this license, visit http://creativecommons.org/ licenses/by/4.0/.

(c) The Author(s) 2020 